\title{
Autonomic Heart Rate Regulation during Mild Dynamic Exercise in Humans: Insights from Respiratory Sinus Arrhythmia
}

\author{
R. SONE ${ }^{\star}$, N. TAN ${ }^{*}$, T. NISHIYASU ${ }^{\dagger}$, and F. YAMAZAKI ${ }^{\ddagger}$ \\ *Department of Exercise and Health Science, Faculty of Education, Yamaguchi University, 1677-1 Yoshida, Yamaguchi \\ City, Yamaguchi, 753-8513 Japan; 'Laboratory of Exercise Physiology, Institute of Health and Sport Sciences, University \\ of Tsukuba, Tsukuba, 305-8574 Japan; and ₹Department of Clinical Pathophysiology, School of Health Sciences, \\ University of Occupational and Environmental Health, Kitakyushu, 807-8555 Japan
}

\begin{abstract}
To better understand the neural mechanism of heart rate $(\mathrm{HR})$ regulation during dynamic exercise, the responses of $\mathrm{HR}$ and the magnitude of respiratory $R-R$ interval variation were examined during exercise and recovery at mild intensities in humans. Eight subjects performed 3-min constant load cycle exercises in a semi-supine position at work rates of 25,50 , and $100 \mathrm{~W}$. The respiratory interval was fixed at $4 \mathrm{~s}$. Peak-to-valley variation in R-R interval caused by respiration was measured breath-by-breath and standardized for tidal volume ( $\Delta R R s t$, a noninvasive index of the degree of parasympathetic cardiac control). At all work rates the HR increased significantly from $2.5 \mathrm{~s}$ after the beginning of exercise $(p<0.05)$ and decreased temporarily and slightly at around $15 \mathrm{~s}$, and the $\Delta$ RRst varied almost inversely. The HR and the $\Delta$ RRst until 12.5 $s$ after the beginning of exercise changed independently of work rate (ANOVA, $p=0.27$ and $p=$
\end{abstract}

0.08). The $H R-\Delta R R s t$ relationship at the initial phase of exercise (for $12.5 \mathrm{~s}$ ) was almost the same at all work rates. These results suggest that the initial HR response to exercise is strongly parasympathetically regulated independently of work rate. The HR recovered slower than the $\Delta R R s t$ at 50 and $100 \mathrm{~W}$. On the $\mathrm{HR}-\Delta$ RRst relationship, the HR during recovery was significantly higher than during exercise at $1 / 3,1 / 2$, and $2 / 3$ levels of pre-exercise $\Delta$ RRst at 50 and $100 \mathrm{~W}$ and at the $1 / 3$ level at $25 \mathrm{~W}(p<0.05)$. At $25 \mathrm{~W}$, the difference in $\mathrm{HR}$ at the $1 / 3$ level was 5.5 beats $\cdot \mathrm{min}^{-1}$, and the HR increase to exercise was 21.2 beats $\cdot \mathrm{min}^{-1}$. We suggest that a HR regulatory system responds slower than a cardiac parasympathetic system to exercise, a cardiac sympathetic system, is activated even during mild exercise in humans. [The Japanese Journal of Physiology 54: 273-284, 2004]

Key words: respiratory sinus arrhythmia, autonomic nervous system, exercise, recovery after exercise.

\begin{abstract}
The neural mechanism of heart rate (HR) regulation is very critical during dynamic exercise. The mechanism has been examined by the use of such approaches as pharmacological blockade, measurements of plasma norepinephrine concentration ([NE]) and muscle sympathetic activity, and the spectral analysis of R-R interval variability in humans [1-5]. It has been widely considered that the HR is increased almost exclusively by a withdrawal of cardiac parasympathetic activity at mild intensities of exercise, and the HR increase asso-
\end{abstract}

ciated with higher intensities of exercise results from both a withdrawal of cardiac parasympathetic activity and an activation of cardiac sympathetic activity.

Although the pharmacological approach has been frequently used for examining the autonomic HR regulation, there is a possibility that interference with the activity of one division of cardiac autonomic system leads to compensatory changes in the other $[3,6]$. If this were true, a relative contribution of each of the two components to the autonomic HR regulation dur-

Received on December 2, 2003; accepted on May 25, 2004

Correspondence should be addressed to: Ryoko Sone, Department of Exercise and Health Science, Faculty of Education, University of Yamaguchi, 1677-1 Yoshida, Yamaguchi City, Yamaguchi, 753-8513 Japan. Phone/Fax: +81-83-933-5389, E-mail: sone@yamaguchiu.ac.jp 
ing dynamic exercise would not be estimated exactly by the technique. Also, since the response of sympathetic activity differs from organ to organ, the plasma [NE] and muscle sympathetic activity may not necessarily change parallel to the cardiac sympathetic activity $[7,8]$. A previous study employing a spectral analysis of R-R interval variability suggested that cardiac sympathetic activity is activated during mild cycle exercise $(10,20$, and $30 \%$ of nominal maximum) [9]. It has also been observed that cardiac sympathetic activity recorded directly in conscious cats is activated during mild exercise, which includes walking $[7,8]$. The measurement of R-R interval variability has become a widely used tool for noninvasively estimating cardiac autonomic activity. However, it has been reported in some papers that the estimation of cardiac sympathetic activity from the results (such as low frequency power) of spectral analysis of R-R interval variability as performed in the above-mentioned studies $[2,5,9]$ is questionable $[10,11]$. Therefore the autonomic HR regulation during mild dynamic exercise in humans should be re-examined without the use of indexes of cardiac sympathetic activity derived from the R-R interval variability.

The HR increases rapidly at the beginning of exercise. A pharmacological study in humans has suggested that the rapid increase results from a withdrawal of cardiac parasympathetic activity, and cardiac sympathetic activity hardly contributes to HR regulation until $10 \mathrm{~s}$ after the beginning of exercise independently of work rate [1]. Previous studies that examined autonomic HR regulation after exercise by using approaches of pharmacological blockade and plasma [NE] measurement in humans have suggested that the HR is decreased by a rapid reactivation of cardiac parasympathetic activity and a slow withdrawal of cardiac sympathetic activity at different intensities of exercise $[12,13]$. Estimating the relative contribution of cardiac parasympathetic and sympathetic activities to autonomic HR regulation based on the results of pharmacological blockade and plasma [NE] may have the limitations mentioned above. However, the less contribution of cardiac sympathetic activity immediately after the beginning and the end of exercise was suggested in spite of a possible compensatory change of cardiac sympathetic activity caused by parasympathetic blockade. Also, the cardiac sympathetic activity is unable to regulate HR on a beat-bybeat basis and with respiration, whereas the cardiac parasympathetic activity can do these fast HR regulations [14-21]. The HR during mild dynamic exercise would be regulated almost neurally [3]. On these bases, if the cardiac parasympathetic activity (exactly, the degree of cardiac parasympathetic control) and the HR could be determined simultaneously at short time intervals of several seconds during mild exercise and recovery, the autonomic HR regulation during the exercise could be estimated by paying attention to a relationship between the two. That is, in case of a contribution of both cardiac parasympathetic and sympathetic activities to the HR regulation, because the cardiac sympathetic activity, or the HR, would be higher during recovery than during exercise at similar cardiac parasympathetic activities, the relationship between the HR and the cardiac parasympathetic activity during recovery would be upward to that during exercise. On the other hand, in case of a contribution of only the cardiac parasympathetic activity, the relationships during exercise and recovery would be the same.

The cardiac parasympathetic activity can be noninvasively estimated from the magnitude of R-R interval variability related to respiratory rhythmicity (respiratory sinus arrhythmia, RSA) [14, 16, 17, 19, 20]. The magnitude of RSA has often been represented by a high frequency power of R-R interval variability. The complex demodulation method has been developed to measure the high and low frequency powers with a high time resolution, and the time resolution is $<15 \mathrm{~s}$ [22]. A peak-to-valley variation of $\mathrm{R}-\mathrm{R}$ interval caused by respiration, which can be measured breathby-breath (i.e., intervals of several seconds), almost perfectly correlates with the spectral analysis measurement [23] and correlates closely with the degree of cardiac parasympathetic control in humans and dogs $[16,17]$.

In the present study, the magnitude of RSA was measured breath-by-breath during and after constant cycle exercise at mild intensities. The comparison of the relationships between the HR and the magnitude of RSA during exercise and recovery suggested the contribution of cardiac sympathetic activity to the HR regulation during mild exercise.

\section{METHODS}

Subjects. Eight healthy and physically active male university students, taking $>30$ min sustained exercise at least three times per week, participated in the present study. The experimental objectives, protocol, possible discomforts, and risks were explained in detail to the subjects, and they gave their written informed consent. The subjects' average age was $20 \pm$ 0.3 years, height $172 \pm 1.6 \mathrm{~cm}$, and body mass $58 \pm$ $2.2 \mathrm{~kg}$.

Protocol. The subjects performed 3-min constant 
load cycle exercises in a semi-supine position four times at each work rate of 25,50 , and $100 \mathrm{~W}$. Preexercise and recovery periods were taken for $5 \mathrm{~min}$ on a cycle ergometer, and the total experimental time at each work rate was $52 \mathrm{~min}$. Pedaling rate was constant at $60 \mathrm{rpm}$. The HR returned to the pre-exercise level during the recovery period under all experimental conditions. The subjects got off the cycle ergometer and took a rest for about 10 min between the different work rate conditions. The order of work rates was randomized.

The magnitude of RSA depends on respiratory interval (RI) and tidal volume $\left(V_{\mathrm{T}}\right)[24,25]$. Thus in the present study, the subjects maintained their RI at $4 \mathrm{~s}$ (2-s inspiration and 2-s expiration) from 2 min before to $5 \mathrm{~min}$ after the exercise in each of the three trials at each work rate (controlled breath $[\mathrm{CB}]$ condition) and did not control it in one trial (free breath [FB] condition). The 4-s RI was selected for easy breathing throughout the pre-exercise, exercise, and recovery periods. A metronome set at 2 beats $\cdot \mathrm{s}^{-1}$ assisted the subjects in controlling their RI. On the other hand, the $V_{\mathrm{T}}$ was free throughout the experiment. Because the relationship between the magnitude of RSA and the $V_{\mathrm{T}}$ is linear at a constant RI either at rest or during exercise, the magnitude of RSA is standardized by dividing by the concurrent $V_{\mathrm{T}}$ [26-28]. So we standardized the magnitude of RSA for $V_{\mathrm{T}}$ in a similar way after the experiment.

Measurement and data analysis. The respiratory volume curve was measured with a respiratory flowmeter (RF-H, Minato) calibrated beforehand by use of a 2-liter syringe. The dead space resulting from the transducer and breathing mask was $130 \mathrm{ml}$. An electrocardiogram (ECG) from the CM5 lead and the respiratory volume curve were stored on a computer (Macintosh LC630, Apple) via an analog-to-digital converter (LAB stack, Keisoku Giken) at a sampling frequency of $500 \mathrm{~Hz}$ during the experiment for the following analysis.

The R-waves were detected first in each ECG record, and the R-R intervals (interval between the two successive R-waves) were then measured beat-by-beat. The R-R interval data were analyzed according to the moving average technique [29], as we have already described in detail [28]. That is, the raw data were interpolated first at 0.25 -s intervals by the Spline method (Fig. 1a) and successively smoothed by a moving average treatment at the number of observations per breath $(N=16)$ (Fig. 1b). Respiratory variation (Fig. 1c) was

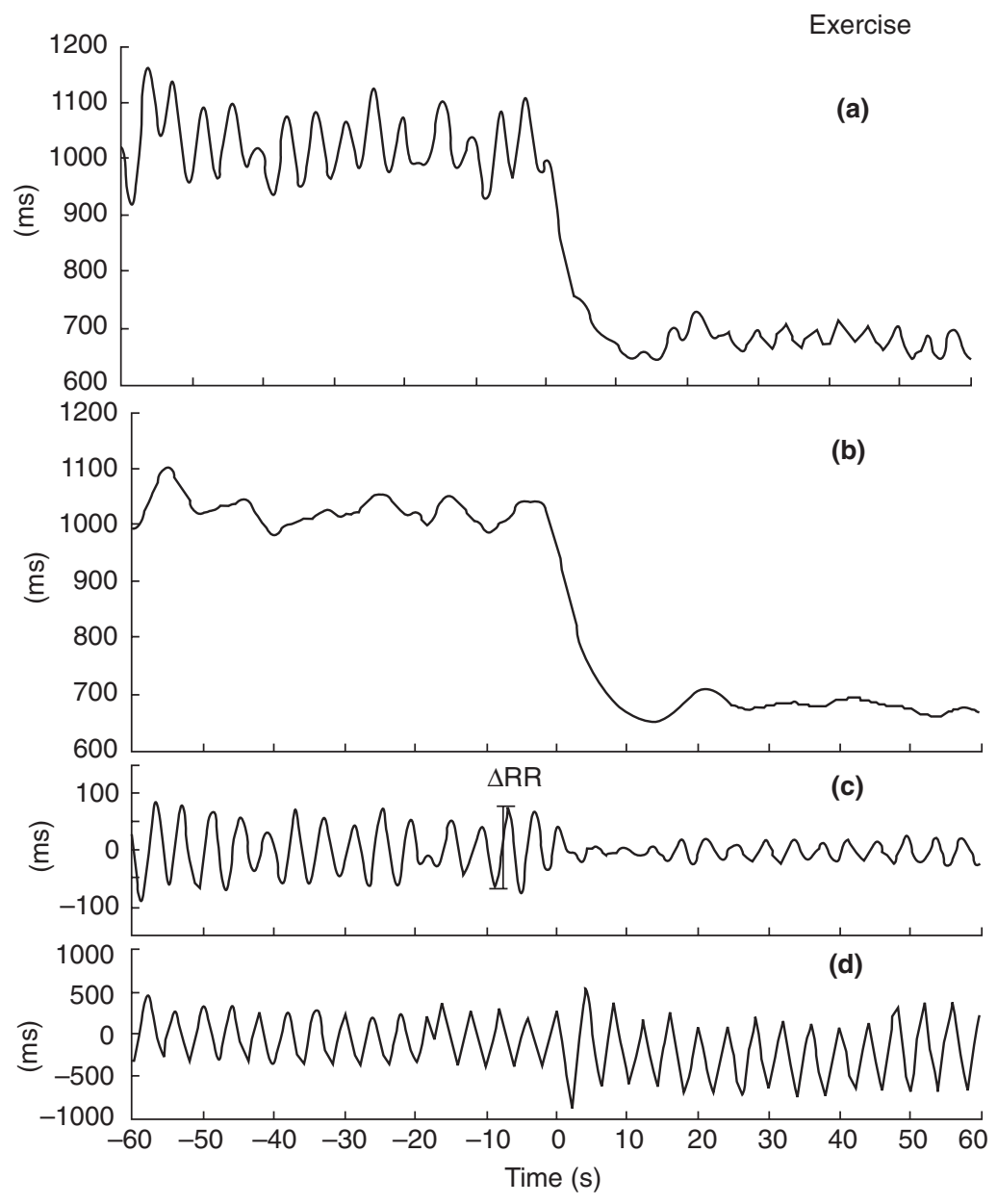

Fig. 1. An example of recorded traces of R$\mathbf{R}$ intervals. (a) Raw data interpolated at $0.25 \mathrm{~s}$ intervals. (b) Slower variability of R-R interval than respiratory variability. This data was obtained use of a smoothing treatment (see Methods). (c) The respiratory variability obtained by subtracting (b) from (a). $\Delta R R$ is the magnitude of respiratory variation. (d) Respiratory volume curve. 
derived by subtracting the smoothed data (b) from the original data (a). The peak-to-valley difference of the respiratory variation ( $\Delta \mathrm{RR})$ was measured breath-bybreath as a reference of the respiratory volume curve and divided by the concurrent $V_{\mathrm{T}}$ for the standardization $(\Delta R R s t)$. The HR calculated from the raw $R-R$ interval data was averaged breath-by-breath. The RI and $V_{\mathrm{T}}$ were measured breath-by-breath from the respiratory volume curve, and minute ventilation $\left(\dot{V}_{\mathrm{E}}\right)$ was calculated. Three values obtained in repeated trials under the CB condition were superimposed and averaged at 5-s intervals for each variable at each work rate. The data under the FB condition were also averaged at the intervals.

The HR changes rapidly at the beginning and the end of exercise. In such cases, whether the respiratory variation in R-R interval could be derived exactly by use of the moving average technique or not was checked with simulated data. That is, time series data consisting of a mono-exponential curve as a trend and a sinusoidal curve as an oscillating component (that is, respiratory variation) were created at 0.25 -s intervals. The baseline value was mono-exponentially decreased to $600 \mathrm{~ms}\left(100 \mathrm{beats} \cdot \mathrm{min}^{-1}\right)$, from $1000 \mathrm{~ms}(60$ beats $\cdot \min ^{-1}$ ), at a time constant of $6 \mathrm{~s}$ in Fig. $2 \mathrm{~A}-\mathrm{I}$ and A-II then increased to $1000 \mathrm{~ms}$ at a time constant of $15 \mathrm{~s}$ in Fig. 2 B-I and B-II. The time constants were settled by reference to previous studies mathematically described the HR responses at the beginning and the end of exercise $[13,30]$. The amplitude and period of the sinusoidal curve were settled at $50 \mathrm{~ms}(100 \mathrm{~ms}$ in terms of the $\Delta \mathrm{RR}$ ) and $4 \mathrm{~s}$, or the RI in the present study, respectively. The beginning of exponential change was timed to the point at which the sinusoidal curve began to change upward in the A-I and B-I and downward in the A-II and B-II. In the A-I and A-II, or a simulation of the $\mathrm{HR}$ response at the beginning of exercise, the oscillating component was underestimated (A-I) or overestimated (A-II) only at the beginning of exponential change. On the other hand, in the B-I and B-II, or a simulation of the HR response at the end of exercise, the oscillating component could be derived almost exactly. On these bases, the first $\Delta \mathrm{RR}$ after the beginning of exercise was excluded from the abovementioned averaging treatment.

Statistics. Data are reported as means \pm SEM. The average for $1 \mathrm{~min}$ before exercise was calculated as the pre-exercise value for each variable. We compared the RI, $V_{\mathrm{T}}$, and $\dot{V}_{\mathrm{E}}$ between the $\mathrm{CB}$ and FB conditions by using the paired t-test. For the HR and $\Delta$ RRst, the effects of work rate on the values at pre-exercise and at end of exercise (at $2 \mathrm{~min} 57.5 \mathrm{~s}$ ) were examined with a one-way analysis of variance (ANOVA), re- spectively. The effects of time and work rate on the $\mathrm{HR}$ and $\Delta \mathrm{RR}$ st for $12.5 \mathrm{~s}$ from the beginning of exercise were examined with a two-way repeated-measures ANOVA and contrast comparison tests for time factor, respectively. Also, the value during recovery was compared with the pre-exercise value by use of a one-way repeated-measures ANOVA and the contrast comparison tests. At 1/3,1/2, and 2/3 levels of the pre-exercise $\Delta \mathrm{RRst}$, the HR during exercise (HRex), the HR during recovery (HRrec), and the difference between the two $(\Delta \mathrm{HR})$ were measured from the HR $\Delta$ RRst relationship for each subject, on which a straight line was applied from dot-to-dot. The lowest level was determined at almost the minimum at which the comparison among three work rates could be done. The effects of work rate and the $\Delta$ RRst level on the HRex, HRrec, and $\triangle \mathrm{HR}$ were examined by use of a two-way ANOVA and the Fisher test, respectively. The HRex was compared with the HRrec at similar $\Delta \mathrm{RR}$ st by use of the paired $t$-test at each work rate. The statistical significance was taken as $p<0.05$.

\section{RESULTS}

\section{Ventilation and HR responses under con-} trolled and free breath conditions. The pre-exercise RI, $V_{\mathrm{T}}$, and $\dot{V}_{\mathrm{E}}$ were not significantly different between the $\mathrm{CB}$ and $\mathrm{FB}$ conditions. The $\dot{V}_{\mathrm{E}}$ during exercise had a tendency to be smaller under the $\mathrm{CB}$ condition (average from 2 to $3 \mathrm{~min}: 15.7 \pm 0.56,18.6$ \pm 0.51 , and $26.7 \pm 0.77 \mathrm{l} \cdot \mathrm{min}^{-1}$ at 25,50 , and $100 \mathrm{~W}$, respectively) than under the $\mathrm{FB}$ condition $(17.0 \pm 0.42$, $20.7 \pm 0.53$, and $28.8 \pm 0.84 l \cdot \mathrm{min}^{-1}$, respectively), except for the initial phase, and the difference for the average was significant at 50 and $100 \mathrm{~W}(p<0.02)$ (Fig. 3). The shorter RI and the smaller $V_{\mathrm{T}}$ were under the FB condition during exercise $(p<0.05)$. The HR responses under the two conditions were almost the same (Fig. 3).

The HR and the $\Delta$ RRst changed markedly immediately before exercise in one subject. So the following results were shown on the average for the other subjects $(N=7)$.

Time course of HR and DRRst (Fig. 4). The preexercise $H R$ and $\Delta R R$ st were not significantly different among the three work rates $\left(60.8 \pm 2.4\right.$ beats $\cdot \mathrm{min}^{-1}$ and $226.6 \pm 30.4 \mathrm{~ms} \cdot l^{-1}$ at $25 \mathrm{~W}, 59.4 \pm 2.4$ beats $\cdot \mathrm{min}^{-1}$ and $258.2 \pm 46.2 \mathrm{~ms} \cdot \mathrm{l}^{-1}$ at $50 \mathrm{~W}$, and $63.7 \pm 3.4$ beats $\cdot \mathrm{min}^{-1}$ and $188.9 \pm 21.6 \mathrm{~ms} \cdot l^{-1}$ at $\left.100 \mathrm{~W}\right)$. At all work rates, the HR increased significantly from $2.5 \mathrm{~s}$ after the beginning of exercise $(p<0.05)$ and decreased temporarily and slightly at around $15 \mathrm{~s}$, and the $\Delta \mathrm{RRst}$ varied almost inversely. The $H R$ and the $\Delta R R s t$ until 

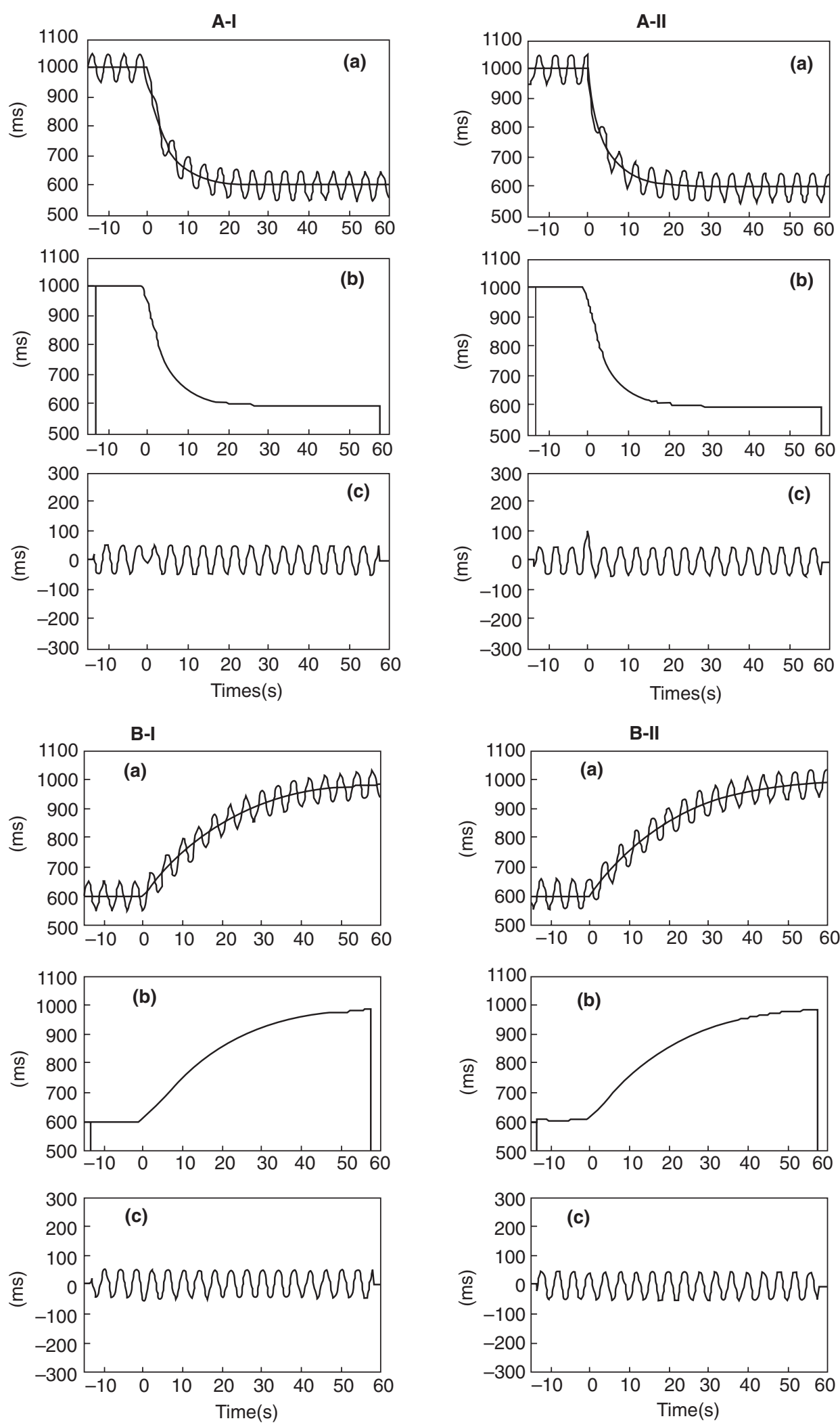

Fig. 2. Derivation of fluctuating component from simulated time series data in $\mathbf{R}-\mathbf{R}$ interval consisting of sine wave and exponential trend at $0.25 \mathrm{~s}$ intervals. When the value begins to exponentially decrease $\mathbf{( A )}$ or increase (B), the sine wave changes upward (I) or downward (II). (a) Simulated time series data (solid line) and exponential trend (dotted line). (b) Smoothed data obtained from the moving average treatment at $N=16$ of (a). (c) Fluctuating component obtained by subtracting (b) from (a). See Methods for more details.

$12.5 \mathrm{~s}$ after the beginning of exercise changed independently of the work rate (ANOVA, $p=0.27$ and $p=$ 0.08 , respectively). A higher HR and a smaller $\Delta$ RRst at the end of exercise were recorded with an increase in work rate $\left(82.0 \pm 3.6\right.$ beats $\cdot \mathrm{min}^{-1}$ and $60.7 \pm 8.0$ $\mathrm{ms} \cdot \mathrm{l}^{-1}$ at $25 \mathrm{~W}, 90.7 \pm 3.1$ beats $\cdot \mathrm{min}^{-1}$ and $35.4 \pm 5.1$ $\mathrm{ms} \cdot l^{-1}$ at $50 \mathrm{~W}$, and $113.0 \pm 4.5$ beats $\cdot \mathrm{min}^{-1}$ and 12.1 $\pm 1.9 \mathrm{~ms} \cdot l^{-1}$ at $100 \mathrm{~W}$, ANOVA, $\left.p<0.01\right)$. The HR during recovery was significantly higher than the preexercise value until $2.5 \mathrm{~s}$ after the end of exercise at $25 \mathrm{~W}, 17.5 \mathrm{~s}$ at $50 \mathrm{~W}$, and $32.5 \mathrm{~s}$ at $100 \mathrm{~W}(p<0.05)$, and the $\Delta$ RRst during recovery was significantly smaller 

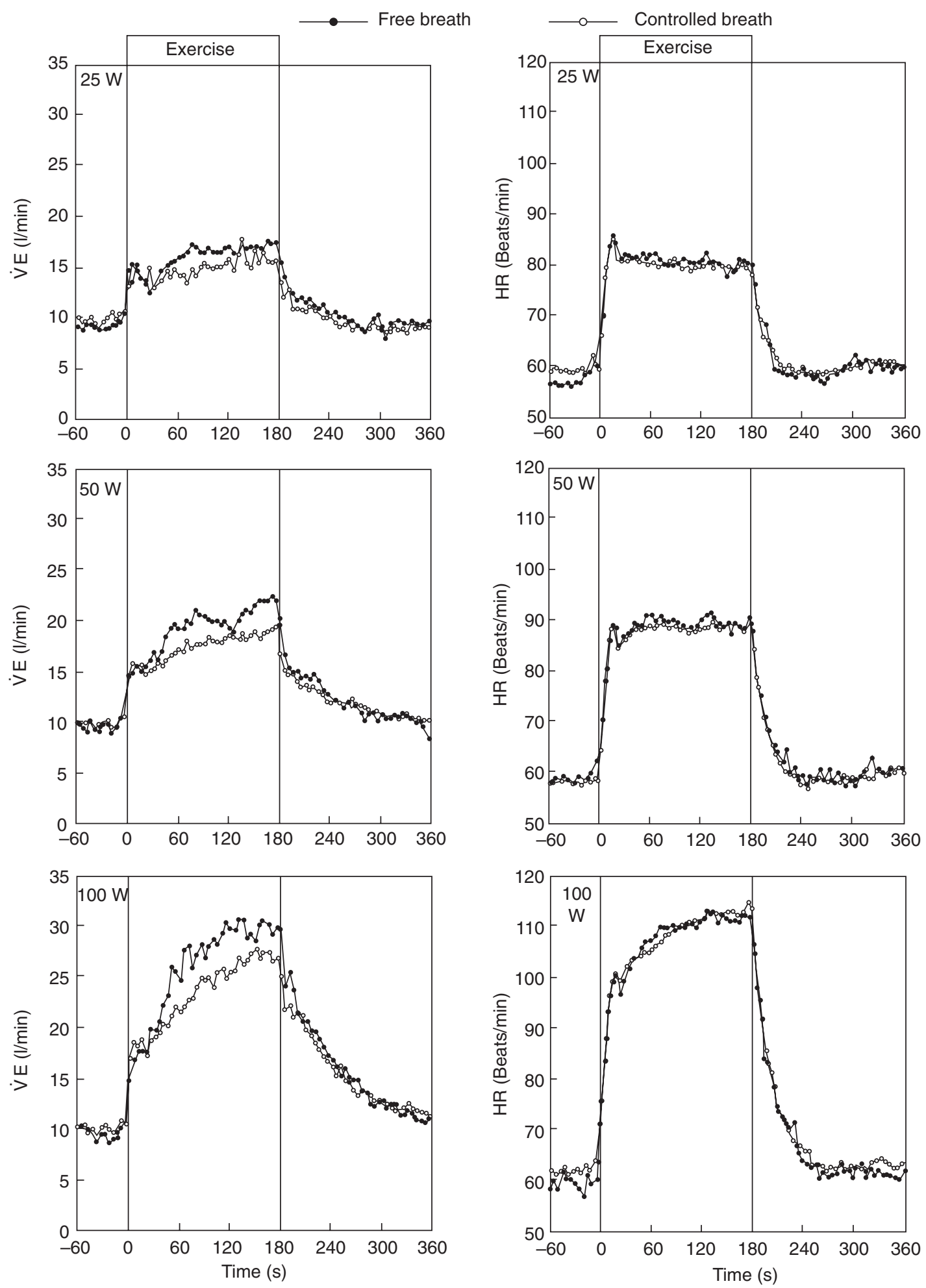

Fig. 3. Ventilation and heart rate responses to exercise under free breathing and controlled breathing conditions. Values are means. $\dot{V}_{E}$, minute ventilation; HR, heart rate

than that at pre-exercise to $2.5 \mathrm{~s}$ at $25 \mathrm{~W}, 12.5 \mathrm{~s}$ at 50 $\mathrm{W}$, and $22.5 \mathrm{~s}$ at $100 \mathrm{~W}(p<0.05)$. Although the $\Delta \mathrm{RRst}$ tended to temporarily become greater than the preexercise value during recovery, the change was not significant.

The HR $-\Delta$ RRst relationships. Figure 5 shows the HR - $\Delta$ RRst relationships from the beginning of exercise (pre-exercise) to $57.5 \mathrm{~s}$ after the end of exercise. The HRex, HRrec, and $\Delta H R$ at the $1 / 3,1 / 2$, and $2 / 3$ levels of the pre-exercise $\Delta$ RRst are shown in Table 1, respectively. The lower HR was certainly at the greater $\Delta$ RRst (ANOVA, $p<0.01$ ). The HRrec was 

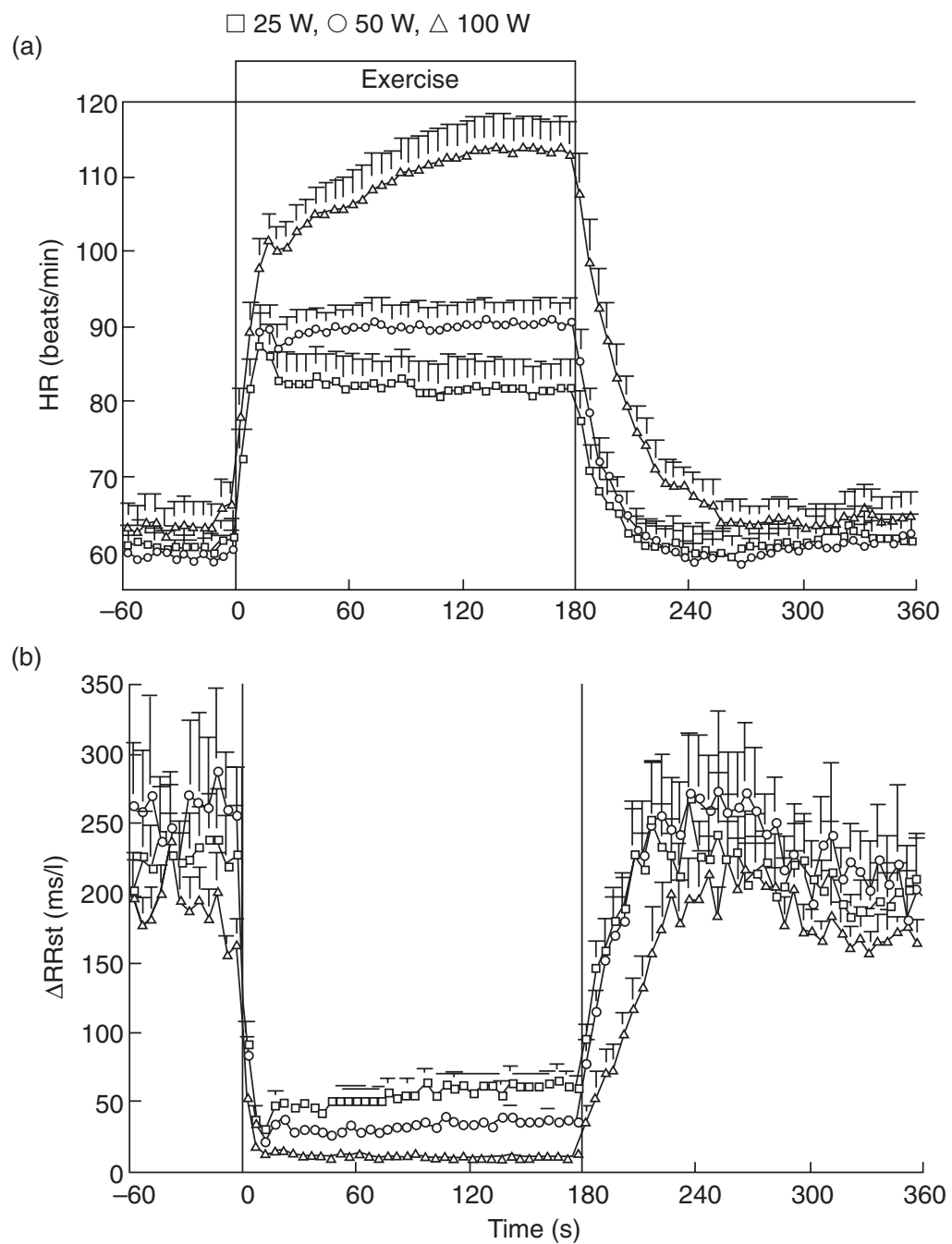

Fig. 4. Time courses of heart rate $(\mathrm{HR})$ and magnitude of respiratory sinus arrhythmia adjusted for tidal volume ( $\Delta$ RRst) during exercise and recovery $(n=7)$. Values are means \pm SEM.

significantly higher than the HRex at the $1 / 3$ level of the pre-exercise $\Delta$ RRst at $25 \mathrm{~W}$ and at all given levels at 50 and $100 \mathrm{~W}(p<0.05)$. The HRex was not affected by the work rate (ANOVA, $p=0.70$ ), and the HR $-\Delta$ RRst relationships for $12.5 \mathrm{~s}$ after the beginning of exercise were certainly independent of work rate. The HRrec was affected by the work rate (ANOVA, $p<0.03)$. The HRrec was significantly higher at 100 $\mathrm{W}$ than at $25 \mathrm{~W}$ at the $1 / 3$ level of the pre-exercise $\Delta$ RRst $(p<0.05)$. The smaller $\Delta$ HR was at the greater $\Delta$ RRst (ANOVA, $p<0.03$ ). The $\Delta$ HR tended to be greater with an increase in work rate, and the $\Delta \mathrm{HR}$ at $100 \mathrm{~W}$ was significantly greater than at $25 \mathrm{~W}$ at the $1 /$ 3 and $2 / 3$ levels of the pre-exercise $\Delta$ RRst $(p<0.05)$.

\section{DISCUSSION}

Changes in respiratory parameters, RI and $V_{\mathrm{T}}$, can seriously confound the association of RSA and cardiac parasympathetic activity $[24,26]$. The subject in the present study therefore controlled the RI at 4 s so that the magnitude of RSA did not change as a result of RI. The breathing technique decreased the $V_{\mathrm{E}}$ during exercise compared with free breathing. The hypoxia and hypercapnia concomitant with the hypoventilation may change the autonomic HR regulation [31, 32]. However, the RI and the $V_{\mathrm{T}}$ during exercise were shorter and smaller under the FB condition than under the $\mathrm{CB}$ condition, respectively, and alveolar ventilation calculated as dead space of 280 $\mathrm{ml}$ (which includes the average physiological dead space, $150 \mathrm{ml}$ ) was not different between the two conditions (FB and $\mathrm{CB}$ conditions; 11.46 and $11.54 l$ at 25 $\mathrm{W}, 14.72$ and $14.40 l$ at $50 \mathrm{~W}$, and 22.49 and $22.54 l$ at $100 \mathrm{~W})$. The HR responses under both conditions were also very similar. Thus it is unlikely that the autonomic HR regulation in the present study became unusual for the application of the breathing technique. The $\Delta \mathrm{RR}$ change caused by the $V_{\mathrm{T}}$ change is probably eliminated by dividing the $V_{\mathrm{T}}$ into the $\Delta \mathrm{RR}$, as the previous studies [26-28] also do. Therefore the magnitude of RSA adjusted for respiration in the present study, $\Delta$ RRst, would more precisely represent the degree of cardiac parasympathetic control. 

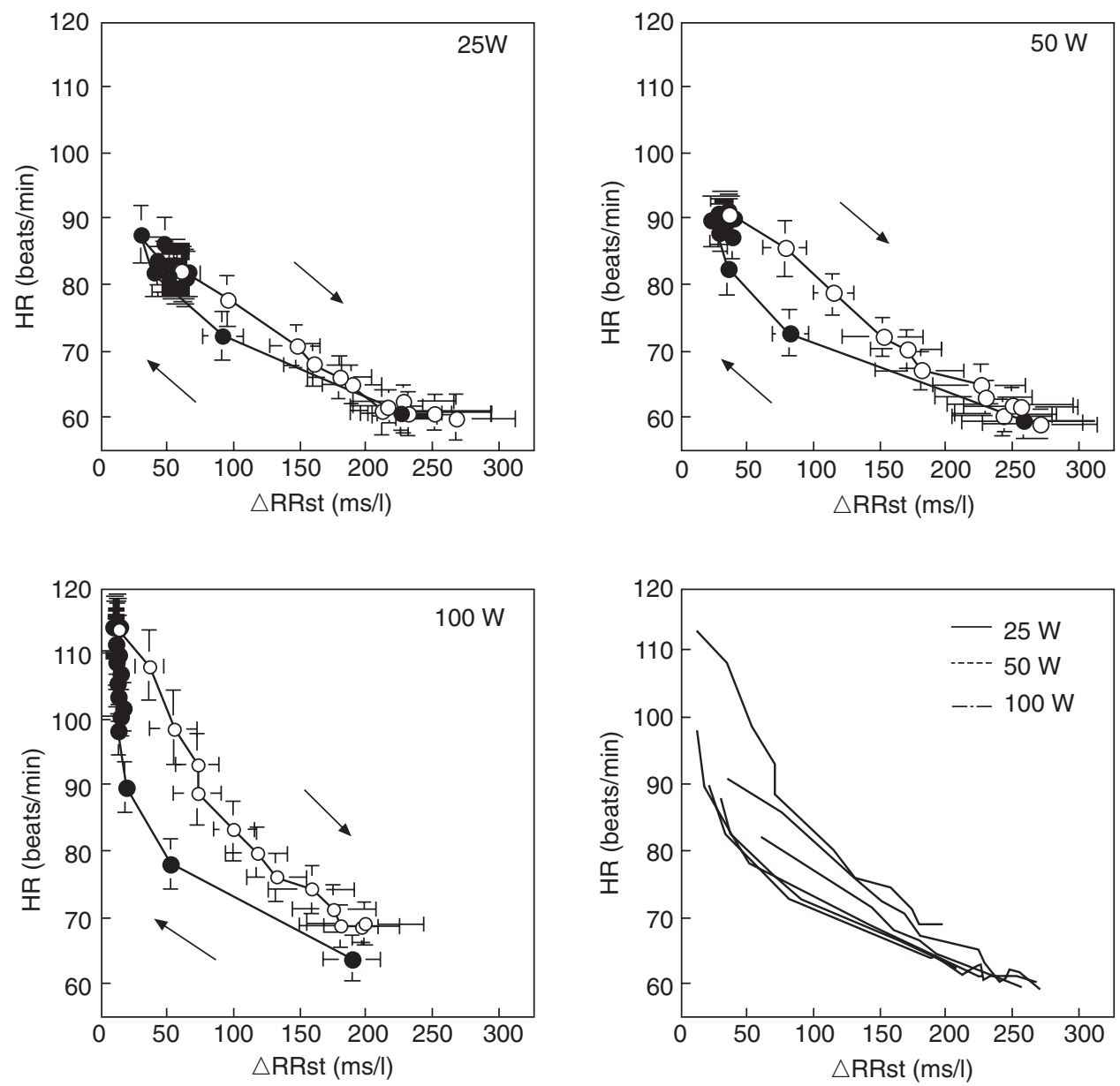

Fig. 5. Relationships between $H R$ and $\Delta R R$ st during exercise and recovery $(n=7)$. Values are means \pm SEM. The filled and white circles present the data plotted at 5-s intervals during exercise (from pre-exercise to $172.5 \mathrm{~s}$ after the beginning of exercise) and recovery (from 2.5 $\mathrm{s}$ before to $57.5 \mathrm{~s}$ after the end of exercise). The bottom right figure shows the relationships during exercise (for only $12.5 \mathrm{~s}$ after the beginning of exercise) and recovery at all work rates. Arrows show the direction of the change of the relationship.

Table 1. Comparison between heart rate during exercise and recovery at similar $\Delta$ RRst.

\begin{tabular}{|c|c|c|c|c|c|c|c|c|c|}
\hline \multirow[t]{2}{*}{ Work Rate } & \multicolumn{3}{|c|}{$\begin{array}{c}\text { HR at } 1 / 3 \text { level of pre-exercise } \\
\Delta \text { RRst } \\
(74.9 \pm 10.2 \mathrm{~ms}) \\
\end{array}$} & \multicolumn{3}{|c|}{$\begin{array}{c}\text { HR at } 1 / 2 \text { level of pre-exercise } \\
\Delta \text { RRst } \\
(112.3 \pm 15.3 \mathrm{~ms})\end{array}$} & \multicolumn{3}{|c|}{$\begin{array}{c}\mathrm{HR} \text { at 2/3 level of pre-exercise } \\
\Delta \text { RRst } \\
(149.7 \pm 20.3 \mathrm{~ms})\end{array}$} \\
\hline & Exercise & Recovery & $\Delta \mathrm{HR}$ & Exercise & Recovery & $\Delta \mathrm{HR}$ & Exercise & Recovery & $\Delta \mathrm{HR}$ \\
\hline $25 \mathrm{~W}$ & $75.5 \pm 2.4$ & $81.0 \pm 3.2^{*}$ & $5.5 \pm 0.8$ & $71.0 \pm 2.5$ & $75.1 \pm 3.7$ & $4.1 \pm 1.8$ & $67.8 \pm 2.3^{\$}$ & $70.4 \pm 2.9^{\$}$ & $2.7 \pm 1.5$ \\
\hline $50 \mathrm{~W}$ & $74.7 \pm 2.6$ & $84.9 \pm 3.4^{*}$ & $10.2 \pm 1.6$ & $71.2 \pm 2.6$ & $79.4 \pm 3.6^{\star}$ & $8.2 \pm 1.8$ & $68.4 \pm 2.4$ & $74.3 \pm 3.8^{*}$ & $5.9 \pm 2.2$ \\
\hline $100 \mathrm{~W}$ & $76.9 \pm 3.2$ & $91.6 \pm 4.1^{\text {*\# }}$ & $14.7 \pm 3.3^{\#}$ & $74.0 \pm 4.1$ & $83.0 \pm 3.7^{*}$ & $9.0 \pm 2.3$ & $68.3 \pm 2.7$ & $76.3 \pm 3.6^{* \$}$ & $8.0 \pm 1.6^{\#}$ \\
\hline
\end{tabular}

Means \pm SEM (beats/min); $\Delta \mathrm{HR}$, difference between the HR during exercise and that during recovery at each case; ${ }^{*}$, vs Exercise; \#, vs 25 W; \$, vs 1/3 level; $p<0.05$.

The rapid increase and the following temporary decrease in HR after the beginning of exercise as observed in the present study have been shown to be abolished by a muscarinic receptor blockade (atropine), but not by a $\beta$-adrenergic receptor blockade, and supposed to result from the rapid withdrawal and the following temporary activation of cardiac parasympathetic activity [31]. A further HR increase after the temporary decrease has been ascribed to increased cardiac sympathetic activity because of the good correlation with plasma [NE] increase [33]. The time course of $\Delta$ RRst during exercise supports the previous observations. Tsuchimochi et al. [8] reported that when conscious cats did treadmill exercise for 1 min at several running speeds corresponding to mild and moderate intensities, cardiac sympathetic activity recorded directly and HR both increased rapidly at the beginning of exercise, and the cardiac sympathetic activity reached the initial peak preceding the initial peak of HR observed at about $11 \mathrm{~s}$ by $3.5 \mathrm{~s}$ at all running speeds. They supposed that the rapid increase in cardiac sympathetic activity contributes to the HR increase at the beginning of dynamic exercise, because a recent study [18] using a sympathetic nerve stimulation protocol to simulate a step increase in [NE] at sinus node indicated that an increase in cardiac sym- 
pathetic activity can produce an increase in HR within a few seconds. However, the cardiac sympathetic activity is unable to regulate HR on a beat-by-beat basis, and slow NE kinetics are the main cause of this behavior [18]. Usually, it also does not contribute to the RSA [15]. These fast HR regulations are done exclusively by the cardiac parasympathetic activity [14-17, 1921]. On these bases, we suggest that the HR immediately after the beginning of exercise is regulated strongly parasympathetically independently of work rate, as also suggested previously [1], and as a result the HR $\Delta$ RRst relationship for $12.5 \mathrm{~s}$ after the beginning of exercise would become similar at all work rates. However, if the cardiac sympathetic activity increased rapidly at the beginning of exercise as shown in cats, the contribution of cardiac sympathetic activity to HR regulation might increase as the time elapsed even at the initial phase of exercise.

The recovery of HR was slower than that of $\Delta R R s t$ at 50 and $100 \mathrm{~W}$, and the HRrec was higher than the HRex at the $1 / 3$ level of the pre-exercise $\Delta$ RRst at 25 $\mathrm{W}$ and at all given levels at 50 and $100 \mathrm{~W}$. These results suggest that HR regulatory systems respond slower than a cardiac parasympathetic system to exercise work during mild exercise.

In heart transplant recipients, the HR responses at the beginning and the end of dynamic exercise are slower than those in healthy subjects, and the magnitude of HR response to exercise is probably dictated only by an increase in plasma catecholamine concentration ([NE] and epinephrine, [E], or their combination, [NE + E]) [34]. So it appears that the HR change may be caused by a mechanical stretch of atrial walls, as a result of increased venous return [35], but is not mainly responsible for the non-neural HR response to exercise. Anyhow, if such slow non-neural HR response occurred during mild exercise, the non-neural component might explain our results. However, both plasma $[\mathrm{NE}]$ and $[\mathrm{E}]$ hardly increase during mild exercise such as $25 \mathrm{~W}$ in healthy subjects $[33,34]$. Furthermore, when in a previously study [36] heart transplant recipients with higher plasma $[\mathrm{NE}+\mathrm{E}]$ at rest and during exercise and with increased sensitivity of myocardial adrenergic receptors in a comparison with healthy subjects exercised at $25 \mathrm{~W}$ for $5 \mathrm{~min}$, the HR increased only about 4 beats $\cdot \min ^{-1}$. Therefore the non-neural HR increase is probably small during mild and shortterm dynamic exercises as performed in the present study, as a pharmacological study also suggests [3].

In conscious cats, the cardiac sympathetic activity changed rapidly preceding the HR at the end of exercise and at the beginning, and as a result, the relationship between HR and the degree of cardiac sympa- thetic activity throughout exercise and recovery showed a higher HR during recovery than during exercise at similar sympathetic activities [8]. The degree of cardiac sympathetic activity is purely per se, whereas the $\Delta$ RRst in the present study represents the degree of cardiac parasympathetic control $[16,17]$. The results of step-like stimulation of cardiac sympathetic nerve show that the process of HR decrease after stimulation is slower than that of HR increase to stimulation [18]. The time course of HR immediately after exercise becomes markedly slow by muscarinic receptor blockade and does not change by $\beta$-adrenergic receptor blockade [12]. Thus even if the cardiac sympathetic activity was withdrawn rapidly after exercise, the HR decrease caused by the withdrawal might be slower than that caused by the reactivation of cardiac parasympathetic activity. As mentioned above, the cardiac parasympathetic activity would start to contribute to the HR regulation faster than the cardiac sympathetic activity at the beginning of exercise. Therefore the HR regulatory system responds slower than cardiac parasympathetic system to exercise that might work during mild exercise is supposed to be the cardiac sympathetic system. This observation is not consistent with those from the pharmacological studies in humans; the sympathetic contribution was hardly observed during a 4-min upright constant load cycle exercise at both 25 and $50 \mathrm{~W}$ and below the HR increase of about 15 and 20 beats. $\mathrm{min}^{-1}$ during supine cycle exercise in a gradual manner every 4 min $[1,3]$. The HRs at the end of exercise at 25 and $50 \mathrm{~W}$ in the present study ( 82.0 and 90.7 beats $\cdot \mathrm{min}^{-1}$, respectively) were also clearly lower than that at which the cardiac sympathetic activity has been supposed to start contributing to the HR regulation during dynamic exercise $\left(100\right.$ beats $\mathrm{min}^{-1}$ ) from the responses of plasma [NE] and muscle sympathetic activity [4].

Pharmacological studies have suggested that the HR regulation during mild exercise is almost exclusively done by the cardiac parasympathetic activity in humans and dogs with high-tonic cardiac parasympathetic activity and little cardiac sympathetic activity at rest $[1,3,37]$, but is also contributed by the cardiac sympathetic activity in rats with significantly tonic activity of both the cardiac parasympathetic and the sympathetic nerves at rest [38]. These different observations are obtained from studies of different species, but they lead us to speculate that the autonomic balance at rest may affect the autonomic HR regulation during exercise. The age and fitness level of subjects and the posture would affect the autonomic balance at rest. Because our subjects were young and had frequently performed endurance exercise and our experi- 
mental posture was semi-supine, these factors would act to favor cardiac parasympathetic predominance [11, 39]. Therefore the inconsistency of observations of the autonomic HR regulation during exercise could not be explained from the pre-exercise autonomic balance.

It has been suggested that in $\operatorname{dogs}$ the cardiac parasympathetic activity estimated from the RSA is withdrawn more strongly during exercise with $\beta$-adrenergic receptor blockade than with no blockade [6]. The $\beta$-adrenergic blockade decreases the HR, and the concomitant withdrawal of cardiac parasympathetic activity must increase the HR. Therefore the results of previous pharmacological studies [1,3] estimating the contribution of cardiac sympathetic activity to the HR regulation during exercise from the difference between the HR with and without $\beta$-adrenergic receptor blockade very likely underestimated the contribution. Also, the response of sympathetic activity differs from organ to organ $[7,8]$. Therefore the response of cardiac sympathetic activity might differ from the responses of plasma [NE] (NE spillover from all sympathetic terminals) and muscle sympathetic activity, and our observation might not be consistent with the previous one [4].

The rapid HR increase at the beginning of exercise is supposed to result from a withdrawal of cardiac parasympathetic activity mainly because of central command [4]. It is also suggested that central command stimulates the cardiac sympathetic system, and the resultant increase in cardiac sympathetic activity contributes to the initial HR increase, based on the results of cardiac sympathetic activity recorded directly during dynamic exercise in conscious cats [7, 8]. It is found that cardiac parasympathetic activity is continuously decreased during passive muscle stretch, the activation of muscle mechanoreceptors, in humans [40] and in decerebrate cats [41]. On the other hand, cardiac sympathetic activity recorded directly in decerebrate cats is increased only at the beginning of exercise [41], but evidence that muscle stretch significantly increases cardiac sympathetic activity is not obtained in humans [40]. On these findings, it appears that this reflex also contributes to the HR increase that occurs during dynamic exercise, mediated mainly by a withdrawal of cardiac parasympathetic activity. The actions of central command and mechano reflex on cardiac parasympathetic activity, cardiac sympathetic activity, and HR must disappear after exercise. Although arterial baroreflex control of HR is reset by exercise and is considered to relate to the HR regulation during exercise, it seems to be a not plausible mechanism leading to the actual HR change $[8,12$,
40, 42]. Metabo-receptor reflex that is sensitive to changes in metabolic environment of muscle would not play a role in the HR regulation during mild and short-term exercise. If the Bainbridge reflex were caused because of increased venous return by exercise, it might contribute to HR increase, but it does not occur markedly in humans [43, 44]. Cardio-pulmonary baroreceptor reflex contributes less to $\mathrm{HR}$ regulation in humans [45]. On these bases, we speculate that the possible increase in cardiac sympathetic activity during mild exercise is mainly produced by the central command mechanism.

The difference between the HR during exercise and recovery at a similar $\Delta \mathrm{RRst}, \Delta \mathrm{HR}$, measured using the $\mathrm{HR}-\Delta \mathrm{RR}$ st relationship at the initial phase of exercise, would roughly correspond to the sympathetic HR increase because the HR at that phase would be increased mainly by a withdrawal of cardiac parasympathetic activity. The time to reach each level of $1 / 3,1 / 2$, and $2 / 3$ pre-exercise $\Delta$ RRst after exercise lengthened with an increase in work rate. Nevertheless, the greater $\Delta \mathrm{HR}$ was observed with an increase in work rate at each level. These observations suggest that the cardiac sympathetic activity increases during exercise dependently of work rate, even if the intensity of exercise is mild. This suggestion is supported by the previous study that directly recorded the cardiac sympathetic activity during mild to moderate exercise in conscious cats [8]. At $25 \mathrm{~W}$, the $\Delta \mathrm{RRst}$ during exercise and recovery reached the $1 / 3$ pre-exercise $\Delta R R s t$ at around $4 \mathrm{~s}$ after the beginning of exercise and immediately after the exercise, respectively. In that case, the $\Delta \mathrm{HR}$ was 5.5 beats $\cdot \mathrm{min}^{-1}$, and the HR increase to exercise was finally 21.2 beats $\cdot \mathrm{min}^{-1}$. Thus it is possible that the cardiac sympathetic activity contributes about a fourth of the HR increase even at a mild intensity of exercise as performed in the present study. If the cardiac sympathetic activity increased correspondingly to the beginning of exercise in humans, as shown in conscious cats [7,8], the resultant HR response might occur with a time delay of several seconds [7, 18, 21]. Consequently, it appears that the cardiac sympathetic activity starts to contribute to the HR regulation within $10 \mathrm{~s}$ after the beginning of exercise, and the $\Delta \mathrm{HR}$ at small $\Delta \mathrm{RR}$ st in the present study underestimated the contribution of cardiac sympathetic activity. For that reason, the $\Delta \mathrm{HR}$ might tend to increase temporarily as the $\Delta \mathrm{RR}$ st increases at all work rates (Fig. 5). Figure 6 shows the $H R-\Delta R R s t$ relationship during exercise (from immediately before to $15 \mathrm{~s}$ after the beginning of exercise) and recovery (from immediately before to $60 \mathrm{~s}$ after the end of exercise) at $25 \mathrm{~W}$ for one subject, using all breath-by-breath data 


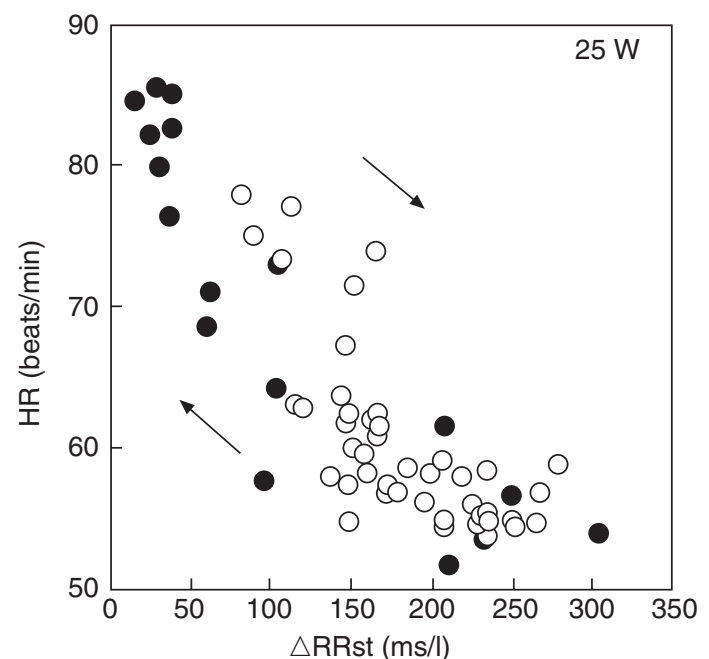

Fig. 6. Relationship between $H R$ and $\Delta R R s t$ during exercise and recovery at $25 \mathrm{~W}$ for one subject, using all breath-by-breath data obtained by three trials. The filled and white circles present the data during exercise (from immediately before to $15 \mathrm{~s}$ after the beginning of exercise) and recovery (from immediately before to $60 \mathrm{~s}$ after the end of exercise). Arrows show the direction of the change of the relationship.

obtained by three trials. From this figure, the higher HR immediately after the end of exercise compared with during exercise at similar $\Delta \mathrm{RRst}$, around $90 \mathrm{~ms} \cdot 1^{-1}$, can be confirmed. But we can't deny the possibility that errors were generated on the process of $\Delta \mathrm{HR}$ measurement because of rough data, especially at the initial phase of exercise.

In the present study, it was suggested that in physically active young subjects the cardiac sympathetic activity contributes to the HR regulation during a 3min constant load cycle exercise even at mild intensity. The autonomic HR regulation during exercise and recovery would be affected by the age, the fitness level of subjects $[11,12,39]$, and the exercise time. In our preliminary experiment, in exercise at a mild intensity for a very short time (12 s) the HR - $\Delta$ RRst relationship was observed to be similar between during exercise and recovery. These points need more consideration. In future investigations, we may need to develop a method to observe the HR $-\Delta$ RRst relationship at higher resolution, e.g., more repetition of exercise, to reduce the errors that may be generated by interpolating the relationship, particularly at the initial phase of exercise.

This study was supported by a grant-in-aid for Scientific Research \#07780070 from the Ministry of Education, Science, Sports and Culture of Japan.

\section{REFERENCES}

1. Maciel BC, Gallo L, Marin Neto JA, Lima Filho EC, and
Martins LEB: Autonomic nervous control of the heart rate during dynamic exercise in normal man. Clin Sci 71: 457-460, 1986

2. Nakamura $Y$, Yamamoto $Y$, and Muraoka I: Autonomic control of heart rate during physical exercise and fractal dimension of heart rate variability. J Appl Physiol 74: 875-881, 1993

3. Robinson BF, Epstein SE, Beiser GD, and Braunwald E: Control of heart rate by the autonomic nervous system. Circ Res 19: 400-411, 1966

4. Rowell LB, and O'Leary DS: Reflex control of the circulation during exercise: chemoreflexes and mechanoreflexes. J Appl Physiol 69: 407-418, 1990

5. Yamamoto Y, Hughson RL, and Peterson JC: Autonomic control of heart rate during exercise studied by heart rate variability spectral analysis. J Appl Physiol 71: 11361142, 1991

6. Billman GE, and Dujardin J-P: Dynamic changes in cardiac vagal tone as measured by time-series analysis. Am J Physiol 258: H896-H902, 1990

7. Matsukawa K: Central control of the cardiovascular system during exercise. In: Exercise, Nutrition, and environmental stress (vol 1), ed. Nose H, Gisolfi CV, Imaizumi K, Cooper Publishing Group, MI, pp 39-64, 2001

8. Tsuchimochi $\mathrm{H}$, Matsukawa $\mathrm{K}$, Komine $\mathrm{H}$, and Murata $\mathrm{J}$ : Direct measurement of cardiac sympathetic efferent nerve activity during dynamic exercise. Am J Physiol 283: H1896-H1906, 2002

9. Lucini D, Trabucchi V, Malliani A, and Pagani M: Analysis of initial autonomic adjustments to moderate exercise in humans. J Hypertens 13: 1660-1663, 1995

10. Brenner IKM, Thomas S, and Shephard RJ: Autonomic regulation of the circulation during exercise and heat exposure. Sports Med 26: 85-99, 1998

11. Carter JB, Banister EW, and Blaber AP: Effect of endurance exercise on autonomic control of heart rate. Sports Med 33: 33-46, 2003

12. Imai K, Sato H, Hori M, Kusuoka H, Ozaki H, Yokoyama $H$, Takeda $H$, Inoue $M$, and Kamada $T$ : Vagally mediated heart rate recovery after exercise is accelerated in athletes but blunted in patients with chronic heart failure. J Am College Cardiol 24: 1529-1535, 1994

13. Perini R, Orizio C, Comande A, Castellano M, Beschi $M$, and Veicsteinas $A$ : Plasma norepinephrine and heart rate dynamics during recovery from submaximal exercise in man. Eur J Appl Physiol 58: 879-883, 1989

14. Akselrod S, Gordon D, Vbel FA, Shannon DC, Barger $A C$, and Cohen RJ: Power spectrum analysis of heart rate fluctuations: a quantitative probe of beat-to-beat cardiovascular control. Science 213: 220-222, 1981

15. Berger RD, Saul JP, and Cohen RJ: Transfer function analysis of autonomic regulation. I. Canine atrial rate response. Am J Physiol 256: H142-H152, 1989

16. Fouad FM, Tarazi RC, Ferrario CM, Fighaly S, and Alicandri C: Assessment of parasympathetic control of heart rate by a noninvasive method. Am J Physiol 246: $\mathrm{H} 838-\mathrm{H} 842,1984$

17. Katona PG, and Jih F: Respiratory sinus arrhythmia: noninvasive measure of parasympathetic cardiac control. J Appl Physiol 39: 801-805, 1975

18. Mokrane A, and Nadeau R: Dynamics of heart rate response to sympathetic nerve stimulation. Am J Physiol 275: H995-H1001, 1998 
19. Pagani M, Lombardi F, Guzzetti S, Rimoldi O, Furlan R, Pizzinelli P, Sandrone G, Malfatto G, Dell'Orto S, Piccaluga E, Turiel M, Baselli G, Cerutti S, and Malliani A: Power spectral analysis of heart rate and arterial pressure variabilities as a marker of sympatho-vagal interaction in man and conscious dog. Circ Res 59: 178-193, 1986

20. Pomeranz B, Macaulay RJB, Caudill MA, Kutz I, Adam D, Gordon D, Kilborn KM, Barger AC, Shannon DC, Cohen RJ, and Benson $\mathrm{H}$ : Assessment of autonomic function in humans by heart rate spectral analysis. Am J Physiol 248: H151-H153, 1985

21. Warner HR, and Russell RO: Effect of combined sympathetic and vagal stimulation on heart rate in the dog. Circ Res 24: 567-573, 1969

22. Hayano J, Taylor JA, Yamada A, Mukai S, Hori R, Asakawa T, Yokoyama K, Watanabe Y, Takata K, and Fujinami T: Continuous assessment of hemodynamic control by complex demodulation of cardiovascular variability. Am J Physiol 264: H1229-H1238, 1993

23. Grossman $P$, and Wientjes C: A comparison of three quantification methods for estimation of respiratory sinus arrhythmia. Psychophysiology 27: 702-714, 1990

24. Hirsch JA, and Bishop B: Respiratory sinus arrhythmia in humans: how breathing pattern modulates heart rate. Am J Physiol 241: H620-H629, 1981

25. Sone R, Fukuoka Y, Yamazaki F, Homma S, and Ikegami $\mathrm{H}$ : Relationship between respiratory period and respiratory cardiac cycle variability: the influence of respiratory blood pressure variability. Jpn J Phys Fitness Sports Med 41: 330-338, 1992

26. Grossman P, Wilhelm FH, and Spoerle M: Respiratory sinus arrhythmia, cardiac vagal control and daily activity. Am J Physiol (in press), 2004

27. Kobayashi H: Normalization of respiratory sinus arrhythmia by factoring in tidal volume. Appl Human Sci 17: 207-213, 1998

28. Sone R, Yamazaki F, Fujii N, Fukuoka Y, and Ikegami H: Respiratory variability in R-R interval during sinusoidal exercise. Eur J Appl Physiol 75: 39-46, 1997

29. Dunstan FDJ: Time series analysis. In: Biological data analysis, ed. Fry JC, Oxford University Press, Oxford, pp 243-289, 1993

30. Feroldi P, Belleri M, Ferretti G, Veicsteinas A: Heart rate overshoot at the beginning of muscle exercise. Eur $\mathrm{J}$ Appl Physiol 65: 8-12, 1992

31. Linnarsson D: Dynamics of pulmonary gas exchange and heart rate changes at start and end of exercise. Acta Physiol Scand 415 [Suppl.]: 1-68, 1974

32. Mauck HP, Jr., Szumski AJ, Forbes JE, Clendenin MA, and Newton RA: Effects of hypercapnea and of hypercapnea in combination with hypoxia on midbrain- induced cardiac dysrhythmias. Am Heart J 92: 315323, 1976

33. Orizio C, Perini R, Comande A, Castellano M, Beschi $M$, and Veicsteinas A: Plasma catecholamines and heart rate at the beginning of muscular exercise in man. Eur J Appl Physiol 57: 644-651, 1988

34. Ferretti G, Marconi C, Achilli G, Caspani E, Fiocchi R, Mamprin F, Gamba A, Ferrazzi P, and Cerretelli P: The heart rate response to exercise and circulating catecholamines in heart transplant recipients. Pflügers Archiv Eur J Physiol 443, 2002

35. Bernardi L, Keller F, Sanders M, Reddy PS, Griffith B, Meno F, Pinsky MR: Respiratory sinus arrhythmia in the denervated human heart. J Appl Physiol 67: 1447-1455, 1989

36. Grassi B, Marconi C, Meyer M, Rieu M, and Cerretelli $P$ : Gas exchange and cardiovascular kinetics with different exercise protocols in heart transplant recipients. J Appl Physiol 82: 1952-1962, 1997

37. O'Leary DS, Rossi NF, and Churchill PC: Substantial cardiac parasympathetic activity exists during heavy dynamic exercise in dogs. Am J Physiol 273: H2135H2140, 1997

38. Overton JM: Influence of autonomic blockade on cardiovascular responses to exercise in rats. J Appl Physiol 75: 155-161, 1993

39. Tulppo MP, Makikallio TH, Seppanen T, Laukkanen RT, and Huikuri HV: Vagal modulation of heart rate during exercise: effects of age and physical fitness. Am J Physiol 274: $\mathrm{H} 424-\mathrm{H} 429,1998$

40. Gladwell VF, and Coote $\mathrm{JH}$ : Heart rate at the onset of muscle contraction and during passive muscle stretch in humans: a role for mechanoreceptors. J Physiol 540: 1095-1102, 2002

41. Murata J, and Matsukawa K: Cardiac vagal and sympathetic efferent discharges are differently modified by stretch of skeletal muscle. Am J Physiol 280: H237H245, 2001

42. O'Leary DS: Heart rate control during exercise by baroreceptors and skeletal muscle afferents. Med Sci Sports Exerc 28: 210-217, 1996

43. Boettcher DH, Zimpfer M, and Vatner SF: Phylogenesis of the Bainbridge reflex. Am J Physiol 242: R244-R246, 1982

44. Takeshita A, Mark AL, Eckberg DL, and Abboud FM: Effect of central venous pressure on arterial baroreflex control of heart rate. Am J Physiol 236: H42-H47, 1979

45. Johnson JM, Rowell LB, Niederberger M, and Eisman MM: Human splanchnic and forearm vasoconstrictor responses to reductions of right atrial and aortic pressures. Circ Res 34: 515-524, 1974 